\title{
Niels Kadritzke Die überraschende Wiederkehr des Nationalismus in die Geschichte
}

\section{Sumpflandschaft nach Tauwetter}

Ein amerikanischer Historiker hat den Abbruch der Berliner Mauer mit dem Fall der Bastille zu Beginn der Franzősischen Revolution verglichen. Diese historische Parallele hat ihn nicht nur fasziniert, sondern auch beunruhigt. Bekanntlich hat Europa im Gefolge der großen Revolution von 1789 eine längere Epoche von Kriegen erleben müssen. Die angebliche Lösung der 'deutschen Frage' wäre bekanntlich ohne die Beendigung des Kalten Krieges nicht möglich gewesen. Doch das Auftauen der vereisten Fronten und die Auflösung des Imperiums, das wir Ostblock nannten, hat noch ganz andere 'nationale Fragen' auf die Tagesordnung gebracht und im Osten und Südosten Europas nationalistische Gefühle und vergessene nationale Konflikte freigesetzt. Überraschend daran nur, daß es so viele überrascht.

Aber selbst wenn uns der Anblick der aufgetauten Sumpflandschaft nationaler Rivalitäten und nationalistischer Exzesse zuweilen melancholisch2 stimmen mag, besteht zur Nostalgie kein Anlaß. Vierzig Jahre Dauerfrost haben gezeigt, daß man Probleme nicht löst, indem man sie einfriert. Ebensowenig läßt sich leugnen, daß die verheißene $»$ Neue Weltordnung* fatal an die alte Balkan-Unordnung von 1914 erinnert. Aus dieser Unordnung wird zwar kein neuer Weltkrieg entspringen, aber regionale Kriege sind wieder führbar geworden. Und zwar im Namen fundamentalistischer Glaubenssätze, die uns kulturell vertrauter sind als der 'fremde' islami-

1 Diesen Text möchte ich dem Andenken eines Freundes und Kollegen widmen. Egon Scotland, Korrespondent der Sïddeutschen Zeitung, starb am 26.7.1991 in Sisak/ Kroatien an den Folgen einer Schußverletzung. An den Folgen des Nationalismus.

2 "Wie wir heute in einem melancholischen Rückblick feststellen können, war es... die große Leistung der kommunistischen Regimes in Vielvölkerstaaten, die verheerenden Auswirkungen des Nationalismus in deren Innerem zu begrenzen.« Und mit Verweis auf Jugoslawien spricht Hobsbawm von der historischen Errungenschaft, daß über * einen längeren Zeitraum als je zuvor in der Geschichte der Region die Völker daran gehindert wurden, sich gegenseitig umzubringen« (Hobsbawm 1990, 207). Schon einige Jahre zuvor gab der Nationalismusforscher Benedict Anderson zu bedenken: *Kalte Krieger, westliche Linke und östliche Dissidenten, die den Rückzug der Roten Armee aus Westeuropa erreichen wollen, sollten sich vergegenwärtigen, daß deren erdrückende Präsenz seit 1945 einen bewaffneten Konflikt zwischen den marxistischen Systemen dieser Region unmöglich gemacht hat. « (Anderson 1983, 12)

Prokla. Zeitschrift fir kritische Sozialwissenschaft, Heft 87, 22. Jg. 1992, Nr. 2, 166-188. 
sche Fundamentalismus, der uns immer wieder als Hauptfeind der Zivilisation und der westlichen Demokratie angetragen wird.

Die fundamentalistischen Glaubenssätze, in deren Namen die Nationalisten aller Länder aufeinander losgehen, sind, wie wir von Eli Kedourie erfahren, keiner uns fremden Religion entsprungen: „Der Nationalismus ist seinen unausgesprochenen Prämissen zufolge eine europäische Doktrin, und seine Ausbreitung in Asien und Afrika folgt der Ausbreitung der europäischen Kultur, getragen vom Prestige und der Selbstgewißheit europäischer Macht. * (Kedourie 1970, 34). Nachdem der alte Kontinent in der ersten Hälfte des 20. Jahrhunderts noch einmal eine Welle 'verspäteter' Nationalismen hervorgebracht hatte, die aggressivste und zugleich irrationalste Strategie dieses 'verspäteten Nationalismus' aber im zweiten Weltkrieg unter ungeheuren Opfern - und mit außereuropäischer Hilfe gestoppt wurde, schien zugleich die engstimige Strategie des Nationalismus als solche erledigt und überwunden. Nach den Grenzbereinigungen der Zwischenkriegszeit und der Fixierung umstrittener Grenzen durch den Kalten Krieg, stand Europa - im Westen wie im Osten - ganz im Zeichen übernationaler Zusammenschlüsse. Nationale Parolen und Irredenta-Ambititonen gehörten im Bewußtsein der meisten Europäer einer vergangenen und abgeschlossenene Epoche an. Auf die Rückkehr dieser Vergangenheit in die Gegenwart reagiert die 'aufgeklärte europäische Öffentlichkeit' erschrocken oder mit beschwörenden Abwehrgesten. Eine Erklärung für die Renaissance nationalistischer Parolen versprechen uns offenbar nur diejenigen, die sie verkünden. Nur ist die Frage nach den Ursachen des Nationalismus viel zu ernst, um die Antwort den Nationalisten zu überlassen.

Die folgenden Überlegungen zum 'neuen Nationalismus' knüpfen an wichtige Erklärungsansätze an, die sämtlich in der englischen Tradition einer pragmatisch argumentierenden und sozialhistorisch gesättigten Theoriebildung stehen. Daß alle drei vorgestellten Ansätze mit signifikantem time-lag ins Deutsche übersetzt wurden, hat verschiedene Gründe. Einer liegt sicher darin, daß sie das Problem des Nationalismus nicht primär vom Extremfall des Nationalsozialismus her angehen.

Ein zweiter Grund dürfte darin liegen, daß alle drei Autoren vehemente Kritiker der marxistischen Theoriebildung sind, der sie jedes analytische Verståndnis für das Nationalismusproblem absprechen. Für Ernest Gellner zum Beispiel reduziert sich die marxistische Nationalismuserklärung auf einen planlosen ideologischen Befreiungsschlag: $\gg$ So wie extreme Schiiten die Ansicht vertreten, der Erzengel Gabriel habe einen Fehler gemacht und die Botschaft Mohammed überliefert, obwohl sie für Ali bestimmt war, so neigen Marxisten zu der Annahme, der Geist der Ge- 
schichte oder das menschliche Bewußtsein habe einen schrecklichen Fehler begangen. Die Erweckungsbotschaft war für Klassen bestimmt, aber durch einen schrecklichen Fehler des Boten wurde sie den Nationen ausgehändigt. Daher müssen die revolutionären Aktivisten heute den falschen Empfänger überzeugen, die Botschaft... an den rechtmäßgen... Empfänger herauszugeben。* (Gellner 1991, 190)

Während Gellner der marxistischen »Theorie der falschen Adresse« mit vitriolischer Ironie begegnet, wirft Benedict Anderson den Marxisten eine schlichte Berührungsangst vor: Da sich der Nationalismus als marxistische Anomalie erwiesen habe, seien sie dem Problem einfach ausgewichen (vgl. Anderson 1988, 13). Tom Nairn schließlich beginnt seine Betrachtungen über die historische Zählebigkeit des Nationalismus mit dem Satz: "Das Problem einer Theorie des Nationalismus steht für das große historische Versagen des Marxismus.* Und Nairn hält dieses Versagen für verhängnisvoller als alle anderen Schwächen und Leerstellen des Marxismus.

\section{Marx, Engels und die 'geschichtslosen Völker'}

Diese Aussagen dreier herausragender Nationalismusforscher lassen sich nicht mit dem Hinweis auf ignorante oder opportunistische Adepten der reinen Lehre entschärfen. Sie zielen auf Marx und Engels selbst, die zu den 'nationalen Fragen' ihrer Zeit engagiert und ausführlich Stellung genommen haben. Zum Beispiel bereits im Kommunistischen Manifest. Die Behauptung, daß die Proletarier kein Vaterland kennen, mag freilich als die läßlichste und verständlichste aller Fehleinschåtzungen durchgehen. Und der abschließende Apell 'Proletarier aller Länder vereinigt Euch' läßt bereits erkennen, daß die vaterlandslosen Arbeiter immerhin einer dramatischen Ermahnung durch die bürgerliche Intelligenz ihrer jeweiligen Nationen bedürfen.

Nach Ernest Gellner fallen Marx und Engels im Manifest einem ökonomistischen Kurzschluß zum Opfer, indem sie die Bewußtseinsformen aus dem unmittelbaren Produktionsprozeß ableiten. Interessanter scheint mir die Frage, wo ihre eigenen Bewußtseinsformen herrühren, wenn sie sich über die Nationalitätenkonflikte ihrer Epoche äußern. Mit anderen Worten: Wie vaterlandslos waren die Väter der Theorie vom vaterlandslosen Proletariat? Für den Engels des Jahres 1849 ist die Antwort eindeutig. Aus seiner Sicht haben sich die europäischen Nationen in zwei politische Lager geschieden: Auf der einen Seite die revolutionären Nationen der Deutschen, der Ungarn und der Polen. Auf der anderen Seite die 'von Natur konterrevolutionåren Völker', die sich von 'der Reaktion' einspan- 
nen lassen. So einfach ist das: die zivilisierten Nationen gegen die barbarischen Völkerschaften, zu denen die Dänen, Basken und Bretonen gehören, vor allem aber die Slawen der Habsburgermonarchie, also Tschechen, Slowenen, Kroaten und Serben. (MEW 6, 172f. und 279)

Im Gewande Hegels und mit dem Furor des Weltgeistes empört sich Engels über all die lästigen 'Natiönchen', die der Industrialisierung, dem Fortschritt, der Revolution im Wege stehen. Er nennt sie 'Völkerabfälle' und macht kein Geheimnis daraus, daß Abfälle auf den Müllhaufen der Geschichte gehören: Der nächste Weltkrieg wird nicht nur die reaktionären Klassen und Dynastien, er wird auch ganze reaktionäre Völker vom Erboden verschwinden machen. Und das ist auch ein Fortschritt.* (MEW 6, 172) Aus welchen historisch-gesellschaftlichen Bedingungen nationale Bewegungen entstehen, welche Lebensbedingungen sich in ihrem 'reaktionären' Bewußtsein artikulieren, interessiert Engels nicht. Es zählt allein der Maßstab des historischen Fortschritts, und der ist identisch mit dem Recht des Stärkeren. Wenn sich acht Millionen Slawen von vier Millionen Ungarn unterjochen ließen, beweist das für den Sozialdarwinisten Engels nur, *wer lebensfähiger und energischer war, die vielen Slawen oder die wenigen Magyaren*. Dasselbe gilt für die Millionen Wallachen, Slawen und Griechen, die sich jahrhundertelang der osmanischen Herrschaft gebeugt haben (vgl. MEW 6, S.278).

Für das Selbstbestimmungsrecht der Völker hat Engels denn auch nur Hohn und Spott übrig. Freiheit und Unabhängigkeit der Völker sind 'moralische Kategorien', bleiben 'leere Traumgebilde' (MEW 6, 273). In der Geschichte zählen nur 'eherne Rücksichtslosigkeit', die großen Eroberer Alexander, Caesar und Napoleón hätten es bewiesen. Das zeigt sich für Engels nicht nur auf dem Balkan. "Ist es etwa ein Unglück « fragt Engels in seiner Polemik gegen Bakunins Idee eines demokratischen Panslawismus - »daß das herrliche Californien den faulen Mexikanern entrissen ist, die nichts damit zu machen wußten? Daß die energischen Yankees durch die rasche Ausbeutung der dortigen Goldminen die Zirkulationsmittel vermehren,... große Städte schaffen, Dampfschiffahrtverbindungen eröffnen,... den Stillen Ozean erst eigentlich der Zivilisation eröffnen, und zum dritten $\mathrm{Mal}$ in der Geschichte dem Welthandel eine neue Richtung geben werden? Die 'Unabhängigkeit' einiger spanischer Califormier und Texaner mag darunter leiden, die 'Gerechtigkeit' und andere moralische Grundsätze mögen hie und da verletzt sein; aber was gilt das gegen solche weltgeschichtliche (sic!) Tatsachen? (MEW 6, 273f) Engels als Flügeldadjudant des marschierenden Weltgeistes, verachtungsvoll auf die Verlierer zurückblickend, die als Fußkranke des zivilisatorischen Fortschritts zuruckbleiben. Diese Lumpenproletarier unter den Na- 
tionen sind für ihn nur 'Völker ohne Geschichte'. Was besagen soll: Völker, die keine Zukunft haben, es sei denn die, sich den historisch durchsetzungsfähigen Nationen assimilieren zu dürfen. Aus solchen Urteilen, vor allem über die Slawen, spricht überaus vernehmlich der deutsche Bürger, der an die beglückende Mission seiner überlegenen Kultur glaubt: Sollen die Tschechen und Slowenen doch froh sein, von den Deutschen geschluckt zu werden, denen sie ihre Zivilisation verdanken und die sich »die Mühe gegeben..., Handel, Industrie, erträglichen Ackerbau und Bildung bei ihnen einzuführen. * (MEW 6, 277)

Solche Urteile sind keineswegs vormarxisitsche Jugendsünden des 'Schülers' Engels, die ihm sein 'Meister' Marx mit der Zeit noch ausgetrieben hätte. Der eurozentrische Fortschrittsglaube ist auch bei Marx anzutreffen, ganz deutlich etwa in den Schriften über die britische Kolonialpolitik in Indien. Und der einzige Aufstand eines unterdrückten Volkes, den Marx und Engels mit Sympathie verfolgten, interessierte sie weniger der Unterdrückten wegen. Die Unabhängigkeit Irlands war nur deshalb ein wichtiges politisches Ziel, weil sie der entscheidende Hebel zu sein schien, um die soziale Revolution in England in Gang zu setzen. Daß der Haß auf die Völker, die den Fahrplan der Revolution zu stören wagten, zumal bei Engels keine Jugendsünde war, ergibt sich aus einem Brief, den er im reifen Alter von 62 Jahren an Eduard Bernstein schrieb. Dessen Sympathie mit den 'unterdrückten Südslawen' versucht er mit folgenden Argumenten entgegenzutreten: "Wir haben ander Befreiung des westeuropäischen Proletariats mitzuarbeiten und diesem Ziel alles andere unterzuordnen. Und wären die Balkanslawen etc. noch so interessant, sobald ihr Befreiungsdrang mit den Interessen des Proletariats kollidiert, so können sie mir gestohlen werden... Ich bin autoritär genug, die Existenz solcher Naturvölkchen mitten in Europa für einen Anachronismus zu halten. (MEW 35, 279ff.) Und um keinen Zweifel zu lassen, von welchem 'Naturvőlkchen' er am wenigsten hält, faßt Engels sein Urteil über die Bulgaren in dem Ausruf zusammen: *Wo in der Welt finden sie ein solches Sauvolk wieder.«(MEW 35, 282.)

Maræ und Engels waren gewiß überragende Köpfe ihrer Zeit - aber eben ihrer Zeit. Moralische Empörung über ihre kulturellen Vorurteile ist die Kehrseite der Enttäuschung darüber, daß auch sie keine überzeitlichen Wahrheiten entdeckt haben. Diese Enttäuschung kann noch heilsamer wirken, wenn sie uns auf eine warnende Frage bringt. Auf die Frage nämlich, in wieweit unsere eigenen Einschätzung des Nationalismus unserem aktuellen zeitlichen und geographischen Horizont verhaftet bleibt. 


\section{Serajewo oder: geschichtslose Völker machen Geschichte}

Es gibt einen weiteren Grund, es sich mit Engels nicht leicht zu machen. In dem zitierten Brief von 1882 berührt er ein Thema, das höchst aktuell anmutet: "Um der paar Herzegowzen willen einen Weltkrieg entflammen, der 1000 mal mehr Menschen kostet, als in der ganzen Herzegowina wohnen - das ist nicht meine Ansicht von der Politik des Proletariats... Und wenn aus dem Aufstand dieser Burschen ein Weltkrieg zu entbrennen droht, der unsre ganze revolutionäre Situation verdierbt, so můssen sie und ihr Recht auf Viehraub den Interessen des europäischen Proletariats ohne Gnade geopfert werden. (MEW 35, 280ff.)

35 Jahre später war der erste Weltkrieg da. Ausgelöst durch das Attentat einiger 'Burschen', die keine Rücksicht auf den von Engels programmierten Lauf der Geschichte nehmen wollten. (Die Pointe, daß dieser 'konterrevolutionäre' Krieg am Ende das welthistorische 'Abfallprodukt' der russischen Revolution hervorbrachte und damit eine fatale Umpolung der Idee und Moral des revolutionären Marxismus, kann hier nicht weiter erörtert werden.) Zwar waren es keine Viehräuber aus Herzegowina, sondern junge serbische Nationalisten aus Bosnien, die den österreichischen Thronfolger in Serajewo erschossen. Doch sie auch sie wollten demonstrieren, daß sie mit ihrer nationalen Unabhängigkeit nicht mehr auf Aktionen anderer warten wollten: weder auf die våterlichen Reformen einer intelligenteren Wiener Obrigkeit, noch auf die brüderliche Revolution des vaterlandslosen Wiener Proletariats.

Die Attentäter von Serajewo würde man heute als Terroristen bezeichnen, so wie lange Zeit die PLO. Im September 1970 fühlte sich die Welt durch einen Satz des Palästineserführers George Habasch herausgefordert: Es sei seinem Volk ganz egal, ob aus dem Kampf um seine nationalen Rechte ein neuer Weltkrieg entstehe. So war es im STERN zu lesen. Das Zitat war gefälscht - übrigens von demselben Joumalisten, der ein paar Jahre später mit den gefälschten Hitler-Tagebüchern Geschichte machen wollte. Das falsche Habasch-Zitat demonstrierte, daß eine Nationalbewegung sich international isoliert, wenn sie ihre Ziele absolut, also ohne Rủcksicht auf den 'Rest der Welt' zu formulieren wagt.

Engels wäre völlig recht zu geben, wenn militante Nationalisten - damals oder heute - einen Weltkrieg ganz allein auslösen könnten. Das können und konnten sie natürlich nicht. 1914 war der Balkan der entscheidende Krisenherd, aber zur bosnischen Lunte brauchte es das Pulverfaß. Zum Weltkrieg entwickelte sich die Balkankrise, weil auf den Ruinen des osmanischen und des habsburgischen Vielvölkerstaates die europäischen Großmächte um geopolitische und ökonomische Einflußssphären konkur- 
rierten.

Das Ende des Ersten Weltkrieges bedeutete auch das Ende des Habsburger und des osmanischen Reiches. Nur der dritte Vielvolkerstaat hat seine Niederlage überlebt. Das russische Reich wurde nicht zerstiickelt, es mubte lediglich Polen in die erneute nationale Selbständigkeit entlassen. Die Revolution von 1917 rettete den territorialen Bestand des Imperiums, den die Rote Amee gegen die westlichen Siegemächte und ihre weißen Truppen behaupten konnte. Doch damit waren auch die Unabhängigkeitsbestrebungen der nichtrussischen Nationalitäten blockiert.

Auf dem Balkan dagegen war mil der Auflősung der Vielvölkerstaaten der Weg zur endgüligen nationalen Selbstbestimmung frei geworden wenn auch keineswegs frei von fremden Einflüssen. Trotz der von USPråsident Wilson proklamierten 'Neuen Weltordnung', die das Selbstbestimmungsrecht der Völker ermöglichen sollte, war die Versailler Friedenskonferenz eine Fortsetzung der Großmacht-Veranstaltungen des 19. Jahrhunderts, nur daß sich die Zahl der kleineren Klienten-Staaten vermehrt hatte. Daß bei der Festlegung der Nachkriegsgrenzen nicht unbedingt die ethno-historischen Gegebenheiten ausschlaggebend waren, erhellt eine Szene, die Harold Nicolson als Berater des britischen Premierministers Lloyd George bei den Verhandlungen in Sèves miterlebte.

Es geht um die Aufteilung des osmanischen Reiches. Die Politiker haben sich um eine Karte niedergelassen. Als Lloyd George, der die griechischen Ambitionen auf das westliche Kleinasien unterstützt, vom italienischen Außenminister auf die Zone südlich von Izmir angesprochen wird, lehnt er entschieden ab: Oh nein, die können Sie nicht haben, sie ist voller Griechen. * Und er zeigt auf der Karte die griechischen Siedlungsgebiete, die sich die ganze Küste entlang bis nach Osten an die syrische Grenze hinziehen. Nicolson wird blaß. "Oh Gott, nein, flüstert er seinem Chef ins Ohr, da gibt es nicht viele Griechen. * Aber doch, antwortete Lloyd George, "sehen Sie nicht, daß hier alles grin eingezeichnet ist? " Da erst geht Nicolson auf, daß sein Chef die geographische fur eine ethnographische Karte gehalten hat: Das Grün der Ebenen fü Griechen, das Braun der Berge für Türken. Aber der britische AuBenminister geht souverän witzelnd ibber seinen Irrtum hinweg. (Nicolson 1933, 72f.).

Diese wahre Geschichte steht fur die vielen Anekdoten, die zur Verschwörungsfolklore aller Nationalbewegungen geworden sind. Sie belegen - ob sie nun nur wahr oder nur plausibel ersonnen sind - den Verrat der großen Imperien an den kleineren Völkern. Fur die außerbalkanischen Großmächte war es stets komfortabel, den eigenen Anteil an der sogenannten Balkanisierung der vormals osmanisch regierten Regionen zu ver- 
schweigen. Doch auch für die rivalisierenden Balkan-Nationalitären hatte der Hinweis auf die Großmachtinteressen die Funktion, von der Tatsache abzulenken, daß die eigene Nationalbewegung mindestens ebenso erbirtert gegen ihre Nachbarnationen kämpfte.

Schon bevor der Erste Weltkrieg alle vorangegangenen Kriegsschrecken relativierte, hatten die Balkankriege von 1912 und 1913 in aller Welt Ent setzen ausgelöst. Der Grund waren die fürchterlichen Kriegsverbrechen, die von allen Beteiligten an der Zivilbevölkerung begangen wurden. Zumal im zweiten Balkankrieg wurden die griechischen, serbischen, bulgarischen Armeen ganz gezielt zur Vertreibung und Liquidierung 'feindlicher' Bevölkerungsgruppen, eingesetzt, das heißt als ein Instrument ethnischer Flurbereinigung. Dieser national organisierte Terror war keineswegs auf die moslemische Balkanbevölkeung beschränkt, die man mit der osmanischen Staatsgewalt aus Europa hinwegfegen wollte. Der zweite Balkankrieg war der Verteilungskampf der 'christlichen' Siegermächte um die europäischen Gebiete des osmanischen Reiches, und erst in diesem Krieg erreichten die Leiden der Zivilbevölkerung ihren Höhepunkt (vgl. Carnegie-Report 1914.) Ein friedliches Zusammenleben der griechischen und slawischen Bevölkerung im Kampfgebiet konnte man sich danach nicht mehr vorstellen. Das Abschlachten endetete mit dem ersten völkerrechtlich besiegelten Bevölkerungsaustausch der Menschheitsgeschichte zwischen den Nachbarländern Bulgarien und Griechenland.

Hätte man dieses Prinzip auf alle Balkangrenzen angewendet, wäre eine allgemeine Bevölkerungswanderung in Gang gesetzt worden. Weil man nicht Millionen von Menschen umsiedeln konnte, blieben überall Minderheiten auf "feindlichem《 Territorium zuriok, Deshalb versuchten fast alle Balkanstaaten, ihre Grenzen zu revidieren, um 'ihre' Minderheiten heimzuholen. Wo das gelang, war damit nur eine andere Bevölkerungsgruppe zur Minderheit geworden. Was Eli Kedourie für die Entstehung der Nationalstaaten festgestellt hat, galt und gilt erst recht für die Balkanregion: "Der Versuch, die politische Landkarte mit linguistischen Kriterien in Einklang zu bringen, ist zwangsläufig eine blutige und brutale Angelegenheit. «(Kedourie 1970, 35)

Doch die Angelegenheit war nicht nur blutig und brutal, sie war auch unlősbar. Ohne Umsiedlungsprogramme läßt sich nicht eine einzige Grenze auf dem Balkan zu einer ethnographisch sauberen Trennlinie machen. Deshalb leben heute noch Rumånen in der sowjetischen MoldauRepublik, Ungarn in Rumånien, Slowaken in Ungarm, Ungam in Serbien, Serben in Albanien, Albaner in den jugoslawischen Regionen Kosovo und Mazedonien, Griechen in Albanien, Türken in Griechenland und Albanien, Bulgaren in Rumänien, womit wir zum Ausgangspunkt unseres 
Minderheiten-Überblicks zurückgekehrt wären. Der damit keineswegs vollständig ist und nicht einmal die besonders komplizierte Lage innerhalb Jugoslawiens und das besonders komplizierte Mazedonien-Problem berücksichtigt.

\section{Kokoschka oder Modigliani}

Bei Ernest Gellner finden wir diesen militanten Balkan-Nationalismus folgendermaßen gekennzeichnet: -In wütender Rivalität mit åhnlichen Konkurrenten erstrebte er die Vorherrschaft über eine chaotische ethnographische Landkarte mit vielen Dialekten, mit unklaren historischen oder linguistischen oder Herkunsftszugehörigkeiten... mit komplexen und vielfäl-tigen Loyalitäten der Verwandtschaft, des Territoriums und der Religion... In vielen Fällen mußten ganze Bevölkerungen ausgetauscht oder vertrieben, mehr oder weniger zur Assimilation gezwungen und manchmal liquidiert werden, um jene enge Beziehung zwischen Staat und Kultur zu erreichen, die das Wesen des Nationalismus ausmacht. *(Gellner 1991, 150f.)

Unter Berufung auf den montenegrinischen Historiker Plamenatz bezeichnet Gellner diesen militanten Typ als den 'östlichen' Nationalismus. Im Gegensatz zum 'westlichen' Typ könne der nicht im Namen einer entwickelten 'klar definierten und kodifizierten Hochkultur' mit deutlich abgestecktem linguistischem Territorium handeln, sondern habe sich die eigene Hochkultur und das eigene kulturelle Geltungsgebiet erst in kürzerer Zeit erkåmpfen müssen. Gellner will damit nicht sagen, daß die zu frühem Selbstbewußtsein gelangten Hochkulturen einen 'sanften Nationalismus' garantieren - der italienische wie v.a. der der deutsche Faschismus bezeugen das Gegenteil. Doch diese Beispiele würde er als 'Irrwege' bezeichnene, während für den 'östlichen Nationalismus' der aggressive Typ als Normalfall erscheint.

Diese Unterscheidung riecht nach jenem abschåtzigen Urteil ůber die o̊stliche Kultur und Mentalität, das auch in den westlichen Begriff der 'Balkanisierung' eingegangen ist. Bei Gellner jedoch ist ein solches Vorurteil nicht mitgedacht, denn er insistiert darauf, daß der 'östliche' Nationalismus sich nicht etwa aus der besonderen Brutalität eines bestimmten Menschenschlages erkläre, sondern "aus der unausweichlichen Logik der Situation*. Mit einer Sensibilität für die konkreten historischen Bedingungen, die wir in Engels' Vorurteilen über die 'balkanesischen Naturvolkchen' vermissen, kann Gellner nur konstatieren, daß sich das Prinzip des Nationalstaates auf dem Balkan unter sehr viel ungünstigeren Vorzeichen durchsetzen mufte als z.B. in Westeuropa. Die Grausamkeit und die Tra- 
gik liegt also bereits in dem rigosen Homogenitåtsideal beschlossen, das laut Gellner die Geschichte der Nationenbildung in ganz Europa und der entwickelten Welt geprägt habe.

Diese säkulare weltgeschichtliche Durchsetzung des Nationalstaatsprinzips faßt Gellner in seinem Schlußkapitel mit einem der Kunstgeschichte entlehnten Bild zusammen: Für ihn gleicht die europåischen Landkarte vor dem Zeitalter des Nationalismus einem Gemälde von Kokoschka - mit vielfach abgestuften Formen und Farben, ohne klare Flächen und Trennlinien zwischen den ethnisch, religiös und kulturell unterschiedlichen Gemeinschaften, die auf engstem Raum zusammenlebten. Dagegen erinnert die Landkarte nach Durchsetzung des Nationalstaatsprinzips eher an Modigliani: Mit klaren, von einander abgesetzen Flächen und Farben, ohne Schattierungen, Ůbergånge und Überschneidungen.(Gellner 1991, 202f.) Gellner würde ohne weiteres zugeben, daß auch ein Kokoschkagemälde seine Reize hat. Doch die Botschaft seiner Theorie liegt darin, daß Modigliani die moderne, Kokoschka hingegen eine untergegangene Epoche repräsentiere. Homogene Nationalstaaten gehören für ihn zu den Selbstverständlichkeiten des modernen Zeitalters. Der Glaubenssatz aller Nationalisten, daß idealerweise die nationalen und politischen Grenzen trennscharf zur Deckung zu bringen seien, entspricht nach Gellner den objektiven gesellschaftlichen Erfordernissen. Denn die Entwicklung von einer statischen, hierarchisch traditionell geprägten Agrargesellschaft zur arbeitsteiligen industriellen Wachstumsgesellschaft erfordert nicht nur die Entwicklung der Produktivkräfte, sondern auch eine gewisse Standardisierung der Menschen. Damit aus einem organischen Geflecht traditioneller Gemeinschaften eine mobile, alphabetisierte, kulturell homogene Gesellschaft werden kann, müssen die Individuen neu sozialisiert werden. Entscheidend hierfür ist die Fähigkeit zu kontextfreier Kommunikation, welche die allgemeine Schulpflicht und ein vom Staat organisiertes Bildungssystem voraussetzt. ${ }^{3}$

Gellners soziologische Theorie des Nationalstaates fußt auf einer fast en-

3 Gellner 1991, 89. Gellners moderner Mensch ist demnach ein Produkt seiner national organisierten Kultur, ein Mensch ohne Nationalität fast so unvorstellbar wie ein Mensch ohne Schatten. Das scheint übertrieben formauliert, aber sicher ist, daB ein Mensch ohne bescheinigte Nationalität es heutzutage fast unmenschlich schwer hat, Anerkennung zu finden und soziale Wurzeln zu schlagen. Eric Ambler-Leser seien an den verzweifelten Kampf erinner, den der Anglo-Araber Arthur Abdel Simpson um staatliche Ausweispapiere und kulturelle Akzeptanz fuhren muß - und der ihn stets in ebenso illegale wie erfolglose Unternehmungen treibt. Simpson wäre in Gellners soziologischem Modernisierungsmodell der anomische Fall schlechthin. Und sein unfreiwilliger und verdrossener Iniemationalismus repräsentiert das Gegenteil des soliden nationalen BewuBtseins, das Gellner bei den am schärfsten gebeutelten Klassen und natürlich beim moderen Proletariat verzeichnet. 
zyklopädischen Übersicht über die - zumindest europäische - Geschichte. Weil sie eine historisch gesättigte Theorie darstellt, ist sie notwendigerweise pessimistisch. Denn Gellner kann natürlich nicht übersehen, daß die Konkurrenz der Nationen über Leichen geht. Seine nüchterne Rechnung lautet: Die Zahl potentieller Nationen ist mindestens zehnmal größer als die Zahl lebensfähiger Staaten. Bei der Homogenisierung der größeren Nationen zu Nationalstaaten bleiben die kleineren oder verspäteten Nationalitåten auf der Strecke. Sie werden mit sanfter oder mit Zwangsgewalt assimiliert, vertrieben und im Extremfall liquidiert.

In einem Vortrag in Berlin hat Gellner die Nacht- und-Nebel-Dimension des Nationalismus gezeichnet. Mit der Anspielung auf die Nazi-Terminologie will er die Schrecken kenntlich machen, die der Nationalismus für die ethnisch heteronomen Subjekte mit sich bringt. Da die modernen Gesellschaften ihre egalitären Versprechen nur selten einzulösen vermögen, zählen die Mitglieder diskriminierter oder zwangsassimilierter ethnischer Gruppen häufig zu den sozial Deklassierten. Wenn die Bruchlinien zwischen den sozialen Schichten mit den ethnischen Trennlinien zusammenfallen, wird das Bewußtsein nationaler Identität wahrscheinlich verstärkt oder überhaupt erst geweckt.

Das Resultat wird in der Regel wiederum eine neue und separatistische Nationalbewegung sein, die es irgendwann zu ihrem eigenen Staat, ihrer eigenen Grammatik, ihrer eigenen Fluggesellschaft bringen kann. Und am Ende auch zur Unterdrückung ihrer eigenen Minderheiten. Wenn also die empirische Durchsetzung des Nationalprinzips einerseits fast als Naturgesetz gesehen wird, anderseits aber nur auf letztlich blutige Weise erfolgt, stehen wir vor einem zutiefst deprimierenden Befund. Entsprechend resigniert muß Gellner der Annahme Kants zustimmen, daß die Parteilichkeit, also die Tendenz, Annahmen zu den eigenen Gunsten zu machen, die gnundlegende menschliche Schwäche darstellt, aus der sich alle anderen ergeben.* Deshalb kơnne sich auch kein Nationalismus eine selbstreflexive Haltung leisten, denn politische Wirksamkeit wäre beeinträchtigt, "wenn die Nationalisten die Untaten ihrer eignen Nation ebenso sensibel registrierten wie diejenigen, die ihrer eigenen Nation angetan werden.* (Gellner 1991, 9)

Das letzte Zitat Gellners benennt die Lebenslüge der Nationalisten, die in entsetzlicher Reinheit von den national-populistischen Führem verkörpert wird, die im zerfallenden Jugoslawien und auf den Trümmern der Sowjetunion ihre ideologische Hegemonie durchgesetzt haben. Es ist eine alte und immer wieder dieselbe Geschichte: daß die Nationalisten aller Kontinente und aller Zeiten wie die heutigen Führer der Kroaten und Ser- 
ben immer nur die Untaten der anderen Seite wahrnehmen und kein Wahrnehmungsorgan für die Untaten der eigenen Nation besitzen.

\section{Nationalität und $Z$ wang, oder: wie man glückliche Türken macht}

Ein Mensch ohne Nationalitåt mag uns heute so wenig vorstellbar sein wie ein Mensch ohne Schatten. Doch seine konkrete Nationalität ist keine biologische Eigenschaft, ja nicht einmal ein »natürliches Gefühl«. Wäre sie das, bräuchte es keinen Nationalismus. Nach Gellner ist Nationalbewußtsein in aller Regel ein Produkt aus Loyalitåt und Zwang, aus freiwilliger Identifizierung und massivem Anpassungsdruck. Nehmen wir als Beispiel die Türkei, die bei vielen Experten als Prototyp einer erfolgreichen nationalistischen Modernisierung gilt. Die stufenweise Umwandlung des multi-ethnischen osmanischen Reiches in einen Nationalstaat erforderte den Einsatz sämtlicher von Gellner aufgezählten Nacht- und Nebelmethoden. Die Liquidierung der armenischen Minderheit durch die Jungtürken vor dem und im ersten Weltkrieg gehörte ebenso dazu wie die Ausschaltung der griechischen Minderheit. Die wurde durch den griechisch-türkischen Bevölkerungsaustausch von 1923 besiegelt, der zwar Resultat des griechischen Angriffskrieges war, jedoch voll in das Programm der Türkifizierung Kleinasiens paßte, das Atatürk von den Jungtürken übernommen hatte.

Doch die Türkei ist damit 1923 noch keineswegs zu einem homogenen Nationalstaat geworden. Von Nationalgefiuhl konnte etwa bei der traditionellen Bauernbevölkerung überhaupt keine Rede sein. Die Schaffung der türkischen Nation aus den Menschen Anatoliens fiel zusammen mit der Einebnung einer vielfältigen Landschaft von sprachlichen, ethnischen, religiösen Gemeinschaften und Identitäten, die bis heute micht abgeschlossen ist. In Schule, Armee und öffentlich organisiertem Leben läuft ein forciertes Programm, das jede neue Generation zu stolzen Türken sozialisieren, beziehungsweise assimilieren soll. Doch nach ůber vier Generationen türkischer Republik registriert das neueste Handbuch für Kleinasien immer noch fast fünfzig ethnische oder religiöse Minderheiten. (Andrews 1989) Die heimliche Leitlinie der Assimilierungsbestrebungen ist der bekannte Ausspruch des Staatsgründers, der in jeder Amtsstube und in jedem Schulzimmer hångt: "Wie glücklich ist, wer sich Türke nennen kannж, so lautet die exakte Übersetzung, und die ist wichtig. Atatürk hat nåmlich keineswegs formuliert: $\gg$ Wie glücklich, wer Türke istk. Zum Türken wird man ernannt, denn dem Staatsgründer war wohl bewußt, daß die echten ethnischen Türken auf dem türkischen Territorium nur eine Minderheit darstellten. Die Staatsraison fordert allerdings, daß 
sich niemand dieser ehrenvollen Ernennung zum Türken entziehen kann. Wer kein glücklicher Türke sein will, macht sich der nationalen Unzuverlässigkeit verdächtig, wie zum Beispiel die nicht assimilierungswilligen Kurden.

Das wichtigste Mittel nationaler Homogenisierung war die Einführung der lateinischen Schrift und der allgemeinen Schulpflicht, die auch die Macht der Religion zurïckdrängte. Für die Kurden und die anderen Minderheiten bot sich damit die Chance, den Anschluß an die sich modernisierende Gesellschaft zu finden, aber eben nur als Türken. Heute sind Millionen ehemaliger Kurden durch die entwickelte O̊konomie der Westtürkei auch ethnisch integriert worden. Doch wo die Modernisierung nicht gelungen ist, wie im Südosten Anatoliens, hat der türkische Nationalstaat den Kurden nicht viel mehr gebracht, als das Verbot, sich Kurden zu nennen und auf kurdisch alphabetisiert zu werden. In der Türkei ist heute der Prozeß noch immer nicht abgeschlossen, den andere Nationalstaaten in Europa schon viel früher durchlaufen haben. Zum Beispiel Griechenland, dessen nationale Befreiungsbewegung zu Beginn des 19. Jahrhunderts in ganz Europa ungeheuere Sympathien genoß. Die Ideologie dieses edelsten und selbstverständlichsten aller Nationalismen war freilich ein Kunstprodukt intellektueller Auslandsgriechen, das den griechischen Bauern von 1821 ebenso fremd sein mochte, wie den anatolischen Moslems der Nationalismus Atatürks. (vgl. Kitromilides 1989) Das Ansinnen der Auslandsgriechen und ihrer philhellenischen Freunde, den Geist des antiken Griechenland in einem nach-osmanischen Staat auferstehen zu lassen, hat die griechische Gesellschaft bis heute auf tragische Weise überfordert. Und das linguistische Projekt einer ans Altgriechische angelehnten Hochsprache hat keineswegs Gellners kontextfreie Kommunikation ermo̊glicht, sondern dazu beigetragen, die Volksmassen der Elite auszuliefern und von wirksamer politischer Partizipation auszuschließen.

\section{Die Erfindung der Nation}

Imagined communities, 'vorgestellte oder eingebildete Gemeinschaften' heißt der Originaltitel der Untersuchungen von Benedict Anderson über Ursprünge und Ausbreitung des Nationalismus. Die deutsche Ausgabe ist unter dem Titel Die Erfindung der Nation erschienen und wurde damit um eine wichtige Dimension verkürzt. 'Erfindung' benennt lediglich die manipulativen Leistungen der nationalistischen Philologen und Historiker, die das Streben nach dem eigenen Staat mit einem bestenfalls idealisierten, schlimmstenfalls verlogenen Geschichtsbild letitimierten. Anderson aber interessiert sich mindestens ebensosehr für das gesamtgesell- 
schaftliche Bedürfnis nach nationaler Selbstgewißheit und historisch bewiesener Kontinuiät, das in den Köpfen der Menschen oder zumindest der selbstbewußter werdenden Schichten entstehen konnte. Er will die psychologische Empfänglichkeit für die 'imaginierte Gemeinschaft der Nation' aufzeigen, die aus den ideologischen 'Erfindungen' der nationalen Erweckungsbewegungen eine höchst reale und objektiv wirksame politische Waffe macht (S. 18f.). Nach Anderson bedient der Nationalismus nämlich emotionale Bedürfnisse, die in den vornationalen traditionellen Gesellschaften von der Religion erfüllt wurden. Am anschaulichsten wird diese metaphysische Dimension des Nationalgefühls an dem universalen Prototyp aller nationalen Monumente: Für Anderson verweist das 'Grabmal des unbekannten Soldaten' mit seiner Affinität zum Tod als letzter Stufe der Schicksalshierarchie auf die mythische Dimension des Nationalgefuihls. Zwar fordern zuweilen auch nicht-nationalistische Ideologen ihre Anhänger auf, ihr Leben zu riskieren, doch ein Grabmal des unbekannte Marxisten oder ein Mahnmal für gefallene Liberale wåre in der Tat eine ideologische Absurdität. Daß der Nationalismus das Erbe der religiösen Denkweisen im Zeitalter der Aufklärung antreten konnte, erklärt Anderson so: *Mit dem Verfall der Religiosität verschwand das Leid, in das der Glaube eine Ordnung gebracht hatte, keineswegs. Der Zusammenbruch des Paradises macht den Tod willkürlich und überführt jeden Erlösungsgedanken der Absurditåt. Notwendig wurde somit eine Umwandlung des Unausweichlichen in Kontinuität, der Kontinuitåt in Sinn. Nur wenige Dinge sind hierzu geeigneter, als die Nation. Es ist das Wunder des Nationalismus, den Zufall in Schicksal zu verwandeln. (20)

Mit der Entwicklung des europåischen Nationalismus zur neuen säkularen Religion traten die Intellektuellen die Nachfolge der Priester an. Anderson belegt exemplarisch die Rolle der Philologen bei der Codifizierung der einzelnen nationalen Hochsprachen und der Historiker bei der Erfindung einer rational und emotional eingängigen Nationalgeschichte. Als potentielle Konsumenten dieser philologisch-historiographischen Revolution ermittelt er die alten gebildeten Klassen und die neuen bürgerlichen Berufsgruppen. Die Beispiele, die uns Anderson vorführt, weisen oft überraschende Bezige zu unserer unmittelbaren Gegenwart auf. Wir erfahren z.B. daß um 1830 die Sprache der Slowemen, der Serbokroaten und der Bulgaren noch als Dialekte einer slawischen Grundsprache galten und sich erst danach in separate Schriftsprachen auseinander differenzierten. (78) Dieser Prozeß geht bis heute weiter, insofern die slawomazedonische Schriftsprache erst während der letzten 40 Jahre eingefuhrt wurde. Und zwar mix dem ausdrücklich politischen Ziel, den slawophonen Bewohnern des nördlichen Mazedonien ein eigenständiges 
Nationalgefühl gegenüber Serben, Bulgaren und Griechen zu vermitteln. Obwohl diese Sprache in Sofia lediglich als bulgarischer Dialekt gesehen und in Athen sogar heute noch für 'nichtexistent' erklärt wird, ist sie innerhalb weniger Schulgenerationen zu einer unleugbaren Realität geworden, die der Bevölkerung des sůdlichsten jugoslawischen Staates eine wichtige Dimension ihrer eigenständigen Identität erfahrbar macht.

Auch die problematischen Leistungen der nationalen Intelligenz stellen ihre aktuelle Wirksamkeit unter Beweis. So ist die abenteuerliche Erfindung der kroatischen Nationalhistoriker, die dem kroatischen Unabhängigkeitsstreben eine tausendjährige Geschichte erdichtet haben, in der schwülstigen Präambel der kroatischen Verfassung von 1991 zur Staatsdoktrin erhoben worden (Nakarada 1991, 392, Anm. 16). Die serbischen Nationalisten wiederum begründen noch heute die Entrechtung der 90prozentigen Mehrheit von Albanern im Kosovo mit dem nekrophilen Argument, daß hier vor 600 Jahren in der Schlacht auf dem Amselfeld serbisches Blut vergossen wurde.

Anderson schildert den Prozeß der geistigen Modernisierung und Homogenisierung, den Gellner mit seiner Nationalismustheorie idealtypisch entworfen hat. Dabei stößt er immer wieder auf einen historischen Widerspruch, den er für die ambivalenten Konsequenzen nationalistischer Ideologien verantwortlich macht. Die vor- oder übernationalen Dynastien versuchen sich der volkstümlichen Nationalbewegungen zu erwehren, indem sie ihnen einen offiziellen Nationalismus entgegensetzen. Anderson schildert diesen Versuch, die schmale und enge Haut der Nation ủber den riesigen Körper eines Imperiums zu spannen, vor allem am Beispiel der Russifizierung der ethnisch heterogenen Bevölkerung des Zarenreiches (91 u. 113). Allerdings zeigt er die Neigung, die dunklen Seiten des Nationalismus, seine expansionistischen Tendenzen und rassistischen Ausbrüche nur dem dynastisch geprägten offiziellen Nationalismus zuzurechnen und umgekehrt die volkstümlichen Nationalbewegungen als demokratisierende politische Kräfte zu glorifizieren und ihr aggressives und expansives Potential zu verharmlosen.

Dabei versucht Anderson mit Beispielen idyllisch-friedlicher Volkspoesie die These $\mathrm{zu}$ belegen, die eigentliche Botschaft des Nationalismus sei Nächstenliebe, die Liebe zur eigenen nationalen Gemeinschaft, der die interesselose Solidarität ihrer Angehörigen gelte. Nur im Namen einer solchen Gemeinschaft sei es möglich, vom Individuum existentielle Opfer, also auch die Bereitschaft zum Sterben zu fordern (142ff.). Daß im Namen der Nation immer wieder Haß gegen das Fremde schlechthin gefordert wurde, läßt Anderson lediglich als 'Mißbrauch' gelten, wie er jedem ethischen Prinzip zustoßen kőnne. Einen systematischen Zusammen- 
hang zwischen Nationalismus und Rassismus bestreitet er mit dem Hinweis, Nationalisten würden in historisch-schicksalhaften Begriffen denken, Rassisten hingegen von einer immerwährenden Gefährdung des nationalen Erbguts, also von ahistorischen Vorstellungen, ausgehen. Expansionistisches Denken schließlich sei dem Nationalismus gånzlich fremd, denn selbst der glühendste Nationalist wïnsche nicht, daß alle Mitglieder der menschlichen Gattung der eigenen Nation angehören. Derartige Wünsche sind allerdings auch dem glühendsten Rassisten fremd, dena der will sich von der fremden Rasse entweder abgrenzen, oder als Herren-rasse über sie herrschen. Auf dieser abstrakten Ebene läßt sich der Nationalismus schwerlich entlasten. Er muß sich vielmehr - wie jede andere Lehre mit praktischem Anspruch - an seinen konkreten historischen Formen messen lassen.

\section{Where the East begins}

1929 verfaßte Hamilton Fish Armstrong, Journalist und späler Herausgeber der 'Foreign Affairs', eine heute noch lesenswerte Balkanreportage. Das Buch hatte den Titel 'Where the East begins'. Was Armstrong damit sagen wollte, machte er anhand seiner einleitenden Reiseanekdoten deutlich.

"Die Balkanreise beginnt in Triest mit der Erage eines italienischen Kellners: 'Sie fahren nach Zagreb? In diesem Drecknest können es nur diese kroatischen Schweine aushalten; wir hier in Triest, wir halten die Grenze von Dantes Zivilisation. Dahinter, da beginne der Orient.

In Zagreb erläutert ein stolzer Kroate die jahrhundertelange Beziehung seines Volkes zur westlichen Kultur: 'Die Unterdrücker haben immerhin die katholische Religion und das westliche Alphabet hinterlassen. Die Serben dagegen, die haben einfach zu lang unter türkischem Einfluß gelebt. Baelgrad - das gehört nun mal leider schon zam Orient.'"s

In Belgrad kommt Armstrong mit einem serbischen Offizier ins Gespräch: " Sie fahren nach Sofia weiter? Hoffentlich wissen Sie, was Sie da evwartet. Diese Bulgaren sind doch halbe Tataren. Die haben noch vor den Türken den Orient auf den Balkan gebrach, und eigentich leben sie noch heule im Orient.

Im Zug von Sofia nach Istanbul fragt ein bulgarischer Mitreisender: 'Waren Sie schon einmal in Konstantinopel? Da werden Sie zum ersten Mal erleben, was Orient bedeutet!" " (Amstrong 1929, Xlji.)

Die interkulturelle Reise von 1929 läBt sich bis in unsere Zeit fortsetzen. Als sich vor ein paar Jahren meine türkischen Freunde A. und A., die schon lange in Berlin leben, zur Heirat entschlossen, hielten sie es für unnötig, ihre Eltem in Istanbul zu konsultieren. Doch die haben das uiberraschend gelassen hingenommen, und die junge Ehefrau wuBte auch, warum: Die waren froh, daß ich ihnen einen Istanbuler präsentiert habe. 
Meine Mutter hat nur gemeint: 'Hauptsache, Du bringst uns keinen aus Anatolien. '* Der Gegensatz zwischen Orient und Okzident, zwischen Islam und Christentum setzt sich bis in die moderne türkische Gesellschaft fort. Der im Westen Europas erfundene Nationalismus hat eine Wertskala etabliert, die von einem zivilisatorischen West-Ost-Gefälle ausgeht. Daß Nationalisten des Ostens und fundamentalistische religiöse Bewegungen nicht nur des Islams die säkularisierte Wertskala umkehren wollen, ist nur die logische Fortsetzung der im 19. Jahrhundert in Gang gebrachten historischen Dynamik.

In dieser Perspektive erweist sich die aggressive Konkurrenz zwischen den Nationalismen nicht nur als die dunkle Seite, sondern als der historische Kern des Problems. Bei aller liebenswürdigen Unschuld patriotischer Lieder - die Erfindung der Nation stiftet mit der Akreditierung einer heiligen Nationalgeschichte immer auch Werturteile über andere Nationen. Auf dem Balkan haben sie nur länger überdauert als anderswo. Die von Anderson festgestellte Kontinuität von religiösen und nationalistischen Bekenntnissen gilt nicht nur für das Bedürfnis nach mythischer Sinngebung des Lebens, sie gilt auch für das Bedürfnis nach Abwehr und Abwertung des Fremden. Elie Kedourie hat auf diese Kontinuität von religiösem und nationalem Homogenitätsbedürfnis hingewiesen: "Wenn uns die europäischen Erfinder der nationalistischen Doktrin auffordern, die Welt als eine Welt von Nationen wahrzunehmen, die hinsichtlich ihrer Geschichte, ihrer Sprache, ihrer Wünsche und Bestrebungen eine vollkommen homogene Einheit sei, so besteht kein Zweifel daran, daß hier zu einem metaphysischen Prinzip erhoben wurde, was die krude Gerechtigkeitsidee des Augsburger Religionsfriedens von 1555 mit der Formel 'cuius regio, eius religio' ausdrickte. «

Das Prinzip nationaler Homogenität läßt sich, wie auch Ernest Gellner zugeben mußte, nur im günstigsten Fall durch die Assimilierung nationaler Minderheiten realisieren. Im brutaleren, aber immer noch unblutigen Fall muß ein Bevölkerungsaustausch vereinbarl werden. Die gewaltsamste Variante ethnischer Flurbereinigung ist auf die Nacht- und Nebelmethoden der Vertreibung und Vernichtung angewiesen. Oder auf erzwungene Grenzverschiebungen mit bzw. ohne Kriege. Das Ende des Ersten Weltkrieges war auch deshalb nur der Anfang einer Zwischenkriegszeit, weil die vielen nationalen Fragen micht durch eine neue intemationale Friedensordnung aufgehoben oder entschärft wurden. Ohne diese offenen Irredentaprobleme hätte die grohe ökonomische und soziale Krise am Ende der Zwanziger Jahre nicht das Treibhausklima für erneute, besonders aggressive nationalistische Bewegungen erzeugen können.

Wean die Verbrechen 2.B. der kroatischen Ustascha noch 50 Jahre später 
in den Köpfen und Seelen vieler Serben Verbrechen gegenüber Kroaten legitimieren können - auch weil der neue kroatische Staat nicht ein Wort des Bedauerns über die Verbrechen seines Vorläufers kundgetan hat - ist damit ein böses Mentekel an die Wand geschrieben. Denn alles spricht dafür, daß auch die Erinnerung an die Verbrechen von heute 50 Jahre später erneut aktiviert werden kann.

\section{Der moderne Janus}

Obwohl Nationalismus nur in extremen Fällen die physische Vernichtung von Minderheiten propagiert, hält Tom Nairn den Faschismus dennoch für einen logischen Fluchtpunkt nationalistischen Denkens: *Über den Nationalismus theoretisieren, ohne dies zu sehen, ist schlimmer als eine Hamletaufführung ohne den Prinzen von Dänemark. Der Nationalismus ist zwar eine so wandelbare und allgegenwärtige Erscheinung der neueren Geschichte, daß man von keinem eindeutigen Archetypus sprechen kann, aber der Faschismus, in seiner umfassenden historischen Perspektive gesehen, sagt uns mehr über Nationalismus als jedes andere Beispiel.* (Nairn 1978, 27)

Diesen Satz hat Tom Nairn vor fünfzehn Jahren in seinem wichtigen Aufsatz 'Der moderne Janus' geschrieben. Auf deutsch ist er nur noch in Bibliotheken nachzulesen. Das Buch, in dem er übersetzt vorliegt, ist zehn Jahre zu frïh erschienen und längst vom Markt verschwunden. Tom Nairn geht in seinen Überlegungen von dem Widerspruch aus, den er als linker Aktivist der Scottish Nationalist Party besonders schmerzlich empfunden hat. Ganze Generationen von praktizierenden Internationalisten hätten sich für nationalistische Bewegungen in den entferntesten Gegenden engagiert, bei nationalen Parolen in geographisch näher liegenden Regionen jedoch immer nur und voreilig Faschismus gewittert. Gegen diese automatische Ůbung, Nationalisten nach Maßgabe ihrer Feinde oder auch Bündnispartner in gute und böse einzuteilen, besteht Nairn auf der prinzipiellen Ambivalenz jedes Nationalismus: »Die Substanz des Nationalismus als solchem ist immer moralisch, politisch und menschlich zweideutig. Deshalb sind moralisierende Einstellungen zum Nationalismus immer so fruchtlos. Sie halten sich immer nur an eines der beiden Gesichter und wollen nicht wahrhaben, daß diese Gesichter zu ein und demselben Kopf gehören. Der Nationalismus gleicht in diesem Sinne dem alten römischen Gott Janus, der auf den Toren stand und mit dem einen Gesicht nach vorne, mit dem anderen nach hinten blickte. Genauso steht der Nationalismus über der Pforte, die für die menschliche Gesellschaft ins Zeitalter der Moderne führt. Und während sich die Menschheit durch 
diese enge Pforte drångt, muß sie verzweifelt in die Vergangenheit zurïckblicken, um Kraft zu schöpfen für die Feuerprobe der Entwicklung.* (Nairn 1978, 28ff.)

Auch Tom Nairn erklärt den Nationalismus aus einer bestimmten Entwicklungs- oder Modernisierungstheorie. Aber das Irrationale, das ihm anhaftet, ist für ihn nicht, wie für Benedict Anderson, eine harmlose Sache, und seine aggressive Fremdenfeindlichkeit sieht er nicht nur als Reaktion auf innergesellschaftliche Widersprüche und Konflikte, wie es Ernest Gellner tut. Für Nairn entspringt die bedrohliche Aggressionsbereitschaft jedes Nationalismus einem tödlichen Widerspruch, der in der ungleichen Entwicklung der Weltgesellschaft als solcher angelegt ist. Hätte sich die Welt gleichmäßig und harmonisch entwickelt, hätte der Ausdruck nationaler Identität womöglich stets die idyllischen Formen annehmen können, die Anderson als ihr harmonisches Wesen sieht. Doch die nationalen Gesellschaften haben sich unter kriegsähnlichen Bedingungen entwickelt und immer neue, schmerzhafte Widersprüche hervorgebracht. Die ungleiche Entwicklung hat vielen Gesellschaften die traditionellen Strukturen zerstört, ohne tragfähige moderne aufzubauen und damit die vereinheitlichende und sozial stabilisierende Seite kapitalistischen Wachstums zur Gelltung zu bringen.

Wo die chaotische, desorientierende Seite der Modernisierung dominiert, gewinnt der Nationalismus für Nairn eine wichtige Funktion. Er ist eben nicht nur 'falsches Bewußtsein'. Vielmehr vermittelt er die Tröstungen einer gemeinsamen Kultur, die eingebildet sein mag, für das wirkliche Befinden der Menschen aber mehr bedeuten kann, als die rationalen Angebote unserer aufklärerischen Tradition. Der Nationalismus könne unter Umständen die einzige reale, wenn auch nicht materielle Kompensation für die Opfer bieten, die der ungleiche Modernisierungsprozeß den Menschen abfordert, die ihn als soziales Elend und kulturelle Åchtung erfahren. Das materielle Dilemma der Rückständigkeit macht empfänglich für die Tröstungen nationalen Denkens und Empfindens.

Aus diesen Überlegungen folgt für Nairn auch, warum Marx und Engels das Problem des Nationalismus nicht entschlüsseln konnten, sondem noch selbst ein Teil des Problems waren. Als Beobachter der Anfänge der industriellen Revolution unterstellten sie einen quasi linearen welthistorischen Prozel , den sie aus den Verlaufsformen der englischen und westeuropäischen Entwicklung extrapolierten. Ein Marxismus, der diese mechanistischen Vorstellungen der Gründerväter nicht überwindet, demonstriert für Naim die Befangenheit in seiner westlichen Herkunft, seine eurozentrische Deformation. Nur wer mit der ungleichen Entwicklung die tatsåchlichen Beziehungen innerhalb der Menschengattung zur Kenntnis 
nähme, könne die notwendigen Irrtümer der marxistischen Nationalismustheorien materialistisch verstehen und damit der keineswegs unerklärlichen und dämonischen Macht der modernen nationalistischen Bewegungen auf die Spur kommen. (a.a.o., 41ff.)

\section{Kein Ende der Geschichte}

Am Ende des Kalten Krieges hat ein intellektueller Stratege der amerikanischen Regierung im Überschwang des Sieges gleich das Ende der Geschichte ausgerufen. Der Irrtum war rasch aufgeklärt. Spätestens mit Beginn der Golfkrise war erwiesen, daß vom neuen Zeitalter der friedlichen Verwaltung internationaler Konflikte nicht die Rede sein konnte. Der Blockkonfrontation folgte die neue globale Unordnung, den klaren Frontlinien die Fragmentierung regionaler Konfliktkonstellationen; um es mit Gellner zu sagen: auf Modigliani folgte Kokoschka.

Das grandiose Scheitern der vermessenen Idee vom Ende der Geschichte bedeutete freilich keinen Triumph für eine rivalisierende Geschichtsphilosophie, und gewiß nicht für die Vertreter einer kritischen Gesellschaftstheorie. Die neue Weltunordnung dürfte im Gegenteil nur den Pessimismus verstärkt haben, der sich schon immer hinter der verzweifelten Leidenschaft verbarg, die viele eurozentrische Intellektuelle für die Revolutionen am anderen Ende der Welt entwickelten. Diesen heimlichen Pessimismus hat Tom Nairn seinen marxistischen Freunden schon in seinem 'Modern Janus' bescheinigt. Als einer der ersten hat er dabei auf jenes Simbild linker Fortschrittsverzweiflung verwiesen, das seitdem fast zu einer Kultfigur geworden ist. Die Rede ist natürlich von Benjamins Engel der Geschichte, der vom Sturm des Fortschritts in eine ungewisse Zukunft getrieben wird. Anders als der Janus auf den römischen Stadttoren hat dieser Engel nur ein Gesicht, und das schaut unverwandt nach rückwärts, in die Vergangenheit. Der zukunftsblinde Flug des Engels symbolisiert unsere Unfähigkeit, vorauszusehen, wohin der Wind des Fortschritts die menschliche Gattung entführt. Das Einzige, was wir erkennen, sind die Trümmer der Katastrophen, die sich am Rande der Piste unseres Entwicklungsprozesses übereinander türmen.

Wenn wir uns auf dieser Piste heute von den nationalistischen Gespenstern der Vergangenheit eingeholt sehen, ist das gleichwohl eine optische Täuschung. Zwar sind viele dieser Figuren mit traditionellen Gewändern kostümiert, aber das erklärt noch nicht ihre Rückkehr in unsere Gegenwart. Wenn sich die heutigen Serben in ihrem Nationalismus an den kroatischen Faschismus von 1943 erinnert fühlen, oder wenn die aktuelle slowakische Unabhångigkeitsbewegung mit den Parolen des Naziprotek- 
torates von 1938 hantiert, zeigt sich darin tatsächlich die Macht der Vergangenheit. Aber warum die Serben und Kroaten und Slowaken ausgerechnet jetzt von dieser Macht ergriffen werden, erklärt sich nur aus ihrer gegenwårtigen Situation. Die aufgetaute Sumpflandschaft, von der wir am Anfang gesprochen haben, mag uns noch so sehr an die alten Tümpel und Wasserläufe erinnern, entstanden ist sie jedenfalls aufgrund einer Klimaveränderung in der Gegenwart.

Dennoch können wir aus den historischen Entstehungsbedingungen der alten Nationalismen lernen, worauf wir in der Gegenwart achten müssen. Von Gellner haben wir erfahren, daß Nationalitätenkonflikte in der Regel dort virulent bleiben, wo die nationalstaatliche Homogenisierung mißlingt. Doch dieses Mißlingen kann zwei Gründe haben. Die Assimilierung von Minderbeiten kann unvollkommen geblieben oder ganz unterblieben sein. Oder die Modernisierung als solche ist mißlungen, und das hat zu sozialen Konflikten geführt, die unzufriedene Minderheiten auf nationalistische Ziele orientiert.

In den meisten Staaten Ost- und Südosteuropas liegen beide Arten des Mißlingens vor. Und in Ländern wie Rumänien oder Jugoslawien hat es ganz den Anschein, als ob sich beide in ihrer Wirkung nicht nur addieren, sondern multiplizieren. In der Sowjetunion wurde spätestens seit Stalin jene Differenzierung in selbständige Nationalstaaten unterdrückt, die in Südosteuropa mit der Auflösung des osmanischen und des Habsburger Reiches unvermeidlich geworden war. Und im gesamten ehemaligen Ostblock blieb die ethnische Gemengelage der Zwischenkriegszeit weitgehend erhalten, ohne durch demokratische Autonomie- oder Minderheitenregelungen entschärft zu werden. Die gesellschaftliche Entwicklung nach 1945 hat die innerstaatlichen nationalen Konflikte im günstigsten Falle unterdrückt; im schlimmsten Falle wurde das Minderheitenproblem, etwa der Ungarn in Rumänien oder der Türken in Bulgarien zu einem gefährlichen zwischenstaatlichen Konfliktpotential.

Inzwischen hat das Scheitern des staatlich organisierten Reproduktionsmodells in allen Ostblockländern zu einer tiefgreifenden sozio-ökonomischen Krise geführt, die eine überaus fatale Modernisierungsbilanz offenbart. Die alten Sozialstrukturen sind bereits erfolgreich zerrüttet, während die materiellen und sozialen Errungenschaften im Chaos des Übergangs vom Staatssozialismus zur Marktwirtschaft aufgezehrt werden. Das gilt auch für das ehemalige Jugoslawien, wo der ungleiche ökonomische Mißerfolg die historischen Rivalitäten zwischen den Teilrepubliken bis zum Nationalitätenkrieg zugespitzt hat.

Wenn ein vernetztes ökonomisches System in einer so riesigen Region zusammenbricht, steht für ungeheuer viele Menschen nicht nur der ge- 
wohnte Lebensstandard, sondern auch das erworbene Lebensgefühl in Frage. Oder noch mehr, denn die Frage lautet zugleich, wie es überhaupt weitergeht. Die Auflösung des Ostblocks hat die unterdrückten Nationalitätenkonflikte Ost- und Südosteuropas also nicht nur wieder aufgetaut, sondern zusätzlich sozial aufgeheizt. Diesen Menschen steht die fürchterliche Feuerprobe einer Entwicklung bevor, die ihnen mehr abfordert, als sie womöglich aushalten können, denn sie bedeutet zugleich einen radikalen Systemwechsel, ist also ein mühsamer Prozeß und abrupte Umstellung zugleich. Die Pforte der Entwicklung, durch die sich diese Gesellschaften im Übergang zu einer Modernisierung unter kapitalistischem Vorzeichen zwängen müssen, ist so fürchterlich eng, daß die Menschen sich gegenseitig die Luft zu nehmen drohen. Und da das neue System mitmenschliche Härte und Konkurrenz zum Prinzip erhebt, wäre es ein Wunder, wenn diese härtere Konkurrenz der Individuen nicht auch die Vorurteile zwischen den Nationalitäten verschärfen würde. Ganz so, wie es Ernest Gellner als Modell ethnischer Diskriminierung entwickelt hat.

Wir können uns die Sache auch am Bild des Januskopfes klarmachen, der für Tom Nairn die Ambivalenz des Nationalismus symbolisiert. In der historischen Situation dieser Gesellschaften kann das Gesicht, das der Zukunft zugewandt ist, bei aller Anstrengung noch nichts erkennen. Oder das, was es erblickt, ist so erschreckend, daß es lieber die Augen schließt. Es bleibt also nur der Blick zurück, denn allein die Orientierung an der guten alten nationalen Vergangenheit bietet einen erträglichen Anblick. Vor dem Hintergrund einer ungleichen Entwicklung ko̊nnen allerdings nicht nur die Zukunftsängste der Zweifelnden und Verzweifelten einen nationalistischen Ausdruck annehmen. Nationale Unabhängigkeit streben auch gerade die Gebiete an, die sich eine günstige Zukunft ausrechnen, wenn sie sich vor dem sozialen Chaos noch rechtzeitig in Sicherheit bringen. Der Anteil des rette-sich-wer-kann-Separatismus ist etwa im Falle Kroatiens und Sloweniens, aber auch bei den baltischen Sowjetrepubliken nicht zu übersehen.

Wie stark die nationalen Gefühle von sozialen Perspektiven bestimmt sind, läßt sich sozusagen per Gegenprobe an einer der vielen umstrittenen Balkangrenzen aufzeigen. Bis vor kurzem haben die griechischen Nationalisten bei der albanischen Regierung gegen die Unterdrückung ihrer angeblich 400.000 Landsleute im Süden Albaniens protestiert. Seit die Albaner ihre Sůdgrenze geöffnet haben und Tausende albanischer Hellenen nach Griechenland abwandern ließen, hat man die Zahl 400000 von offizieller Seite nicht mehr vernommen. Und die Vorstellung, die vielleicht 150000 Griechen, die es tatsächlich in Albaniera gibt, könnten ihre Wie- 
dervereinigung mit Griechenland einklagen, dürfte bei den meisten Griechen keine Begeisterung, sondem nur whiste Alpträume auslösen

Nationale Begeisterung kann also ziemlich schnell, wenn sie mit dem Interesse am sozialen Besitzstand zu kollidieren droht. Wir kennen es auch aus unserem neuen Deutschland einig Vaterland, in dem wir uns anschicken, die Nationalismustheorie um eine aufschlußreiche empirische Variante zu bereichern. Nachdem wir bereits demonstriert haben, daf es eine Nation auch in zwei Staaten aushäll, führen wir der Welt jetzt vor, daß sich ein Nationalstaat auch zwei Gesellschaften leisten kann. Mit der Verwandlung der nationalen in eine soziale Frage - die des Teilens erworbener Besitzstånde nämlich -, sind wir dabei, die pazifierende Wirkung nationaler Einheit vorzuführen. Denn wer wollte es bestreiten: ein åhnliches soziales Gefalle, wie es auf absehbare Zeit zwischen den beiden deutschen Gesellschaften fortbestehen wird, hätte in ethnisch gemischten Staaten schon längst zum offenen Nationalitätenkonflikt geführt.

\section{Literatur}

Anderson, Benedict (1983): Die Erfindung der Nation, Frankfurt/Main 1988.

Andrews, Peter A. (1989): Ethnic Groups in the Republic of Turkey, Wiesbaden.

Armstrong, Hamilton Fish (1929): Where the East Begins, New York/London.

Camegie-Report (1914): Report of the International Commission to Inquire into the Causes and Conduct of the Balkan Wars, hrsgg. vom Carnegie Endowment for International Peace, Washington D.C.

Cummins, Ian (1980): Marx, Engels, and National Movements, London.

Davis, Horace B. (1978): Toward a Marxist Theory of Nationalism, New York/London.

Engels, Friedrich (1849): Der magyarische Kampf, NRZ vom 13. Januar 1849, in MEW $6,165-176$.

ders.: Der demokratische Panslawismus, NRZ vom 15. Februrar 1849, in MEW 6, 270286.

ders. (1882): Brief an Bernstein yom 22. Februar 1882, in MEW 35, 278-285.

Gellner, Ernest (1983): Nationalismus und Moderne, Berlin 1991.

Hobsbawm, Fric J. (1990): Nationen und Nationalismus, Frankfurt/Main.

Kedourie, Elie (1970): Nationalism in Asia and Africa, London.

Kitromilides, Paschalis M. (1989): 'Imagined Communities' and the Origins of the National Questions in the Balkans, in European Quarterly 19. Jg. 1989, 149-194.

Nairn, Tom (1978): Der moderne Janus, in Nationalismus und Marxismus, Rerlin.

Nakarada, Radmila (1991): The Mystery of Nationalism. The Paramount Case of Yugoslawia, in Millenium, Vol. 20, No. 3, 369-382.

Nicolson, Harold (1933): Peacemaking 1919. London.

Rosdolsky, Roman (1964): Friedrich Engel und das Problem der 'Geschichtslosen Vòlker', in Archiv für Sozialgeschichre N, 1964, 87-282. 